the total number of PN bags used (figure 1). This is despite an increase in the mean number PN referrals from 26/month in 2016 to $39 /$ month in 2020 .

Mean\% standard bag usage has increased from $14.5 \%$ in 2016 to $29.5 \%$ in 2020 (figure 2)

There is minimal change in wastage (figure 3) but wastage varied widely between different specialities (surgery: $2.2 \%$, oncology and haematology: $3.6 \%$ and Paediatric Intensive Care (PICU) 7.3\%).

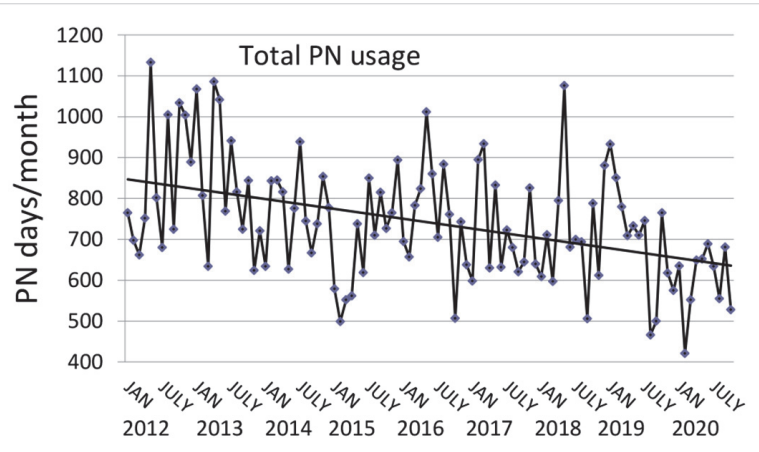

Abstract 09 Figure 1

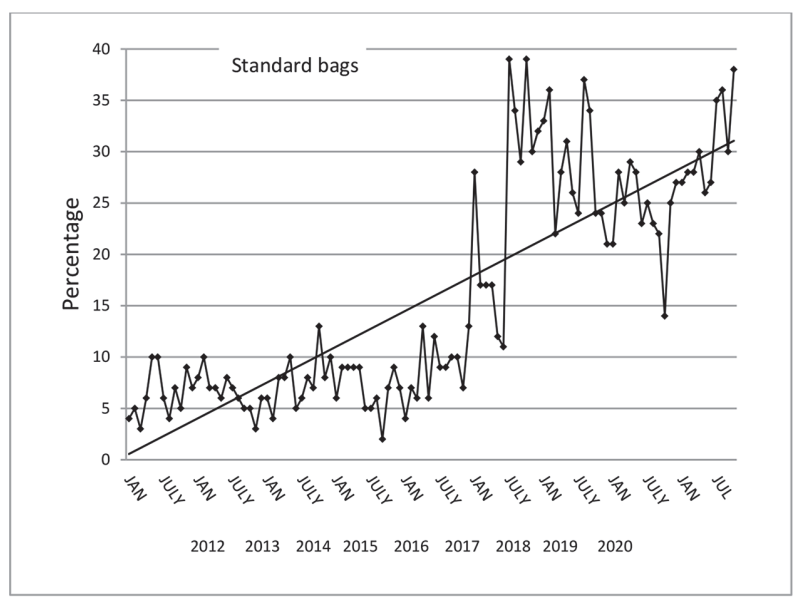

Abstract 09 Figure 2

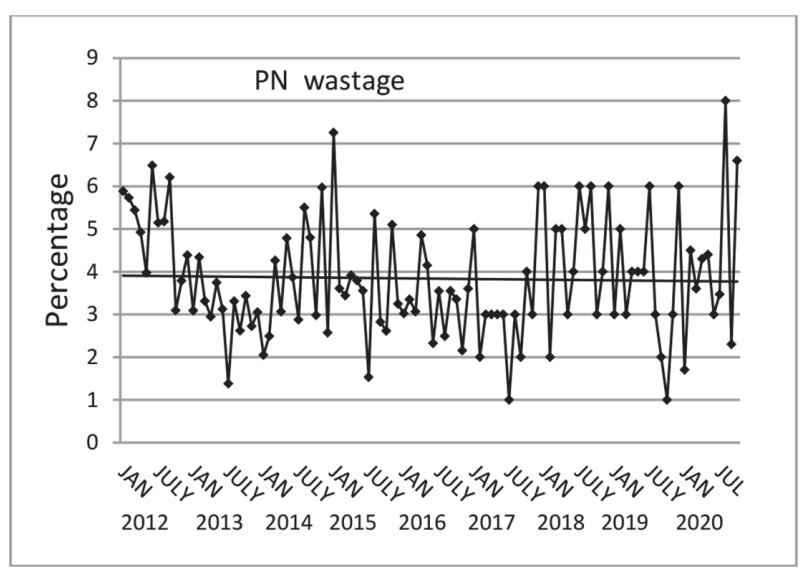

Abstract 09 Figure 3
Summary and conclusion The interventions of the NST have resulted in continued reduction in the number of $\mathrm{PN}$ days despite an increase in referral numbers, suggesting shorter episodes of PN.

A sustained increase in the percentage of standard bags $2017-2020$ is attributed to more frequent consideration of standard PN, particularly when starting and weaning PN. Using clinical expertise the NST has introduced a wider range of standard PN bags. This has also contributed to increased standard PN use without compromising the quality of nutritional support.

There are a number of factors that contribute to reducing PN wastage including using standard bags as these can be recycled. The study has not been able to demonstrate any major impact of standard bag use on overall PN wastage. Wastage is multifactorial and data recording for PN wastage has been optimised to enable thorough evaluation of contributing factors. Most specialities have demonstrated reduced PN wastage, but wastage on PICU remains high. This clinical area will be prioritised for additional NST involvement in 2021.

\section{MAPPING THE CURRENT SERVICE AVAILABILITY IN PAEDIATRIC GASTROENTEROLOGY NETWORKS ACROSS THE UNITED KINGDOM: IS THERE A POSTCODE LOTTERY IN SERVICE PROVISION?}

Sandra Fernandes Lucas, Himadri Chakraborty. Basildon and Thurrock University Hospitals NHS Foundation Trust

\subsection{6/flgastro-2021-bspghan.10}

Background Clinical Networks are formed by Network Centres (NC) and Lead Specialist Centres (LSC) for Gastroenterology and Nutrition to provide high quality, specialist care to the local population. According to the Quality Standards released by RCPCH and BSPGHAN, by definition Network Centres have at least one Consultant Paediatrician with a Special Interest in (SPIN) Paediatric Gastroenterology. It is important to understand the layout of service availability in secondary care and its uniformity across the United Kingdom (UK) to ensure delivery of high-quality care.

Objectives There is scarcity of information about the secondary care network for Paediatric Gastroenterology in the UK. The primary aim of the project was to quantify the secondary care trusts with at least one General Paediatrician with a Special Interest in Gastroenterology and comprehend how these specialists are distributed across the UK. Secondly, we aimed to look at the support these hospitals receive from Lead Specialist Centres in the form of outreach clinics. Finally, the last goal was to map the Paediatric Gastroenterology Clinical Networks in the UK.

Methods The list of hospitals providing Paediatric services in the UK was collected from the National Health Care systems' and Paediatric deaneries' websites for England, Wales, Northern Ireland and Scotland. We identified a number of centres providing Paediatric secondary and tertiary care services, and subsequently contacted them via telephone or e-mail. All the data was collected from Paediatric Doctors (Specialty Registrars or Consultants), Specialist Nurses or Secretaries working in Paediatrics over the period of one year, through standard verbal questionnaire or electronic survey.

Results We identified a total of 153 secondary care trusts providing specialist services to the Paediatric population 


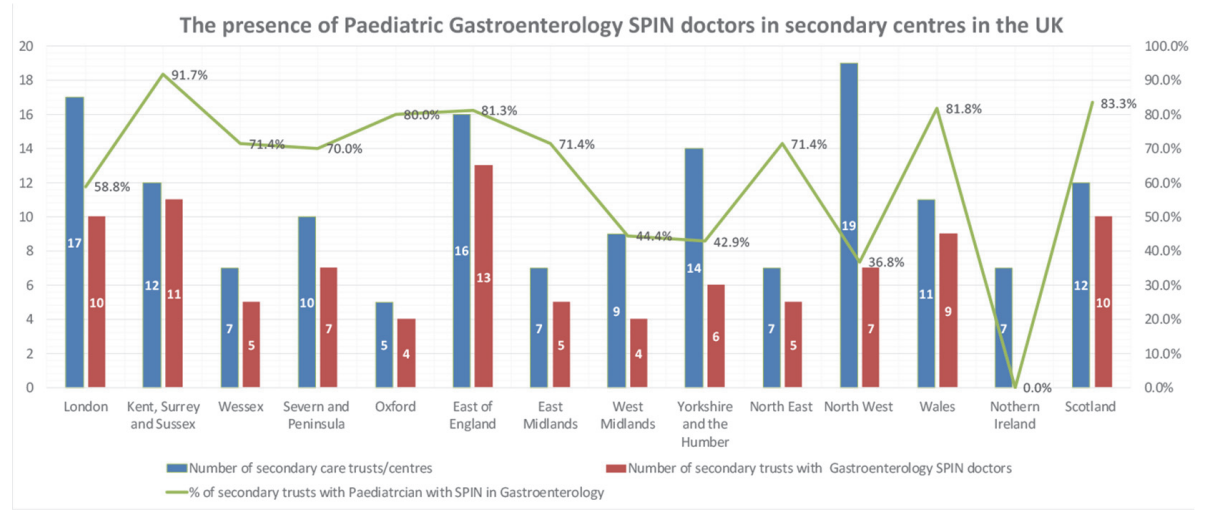

Abstract 010 Figure 1

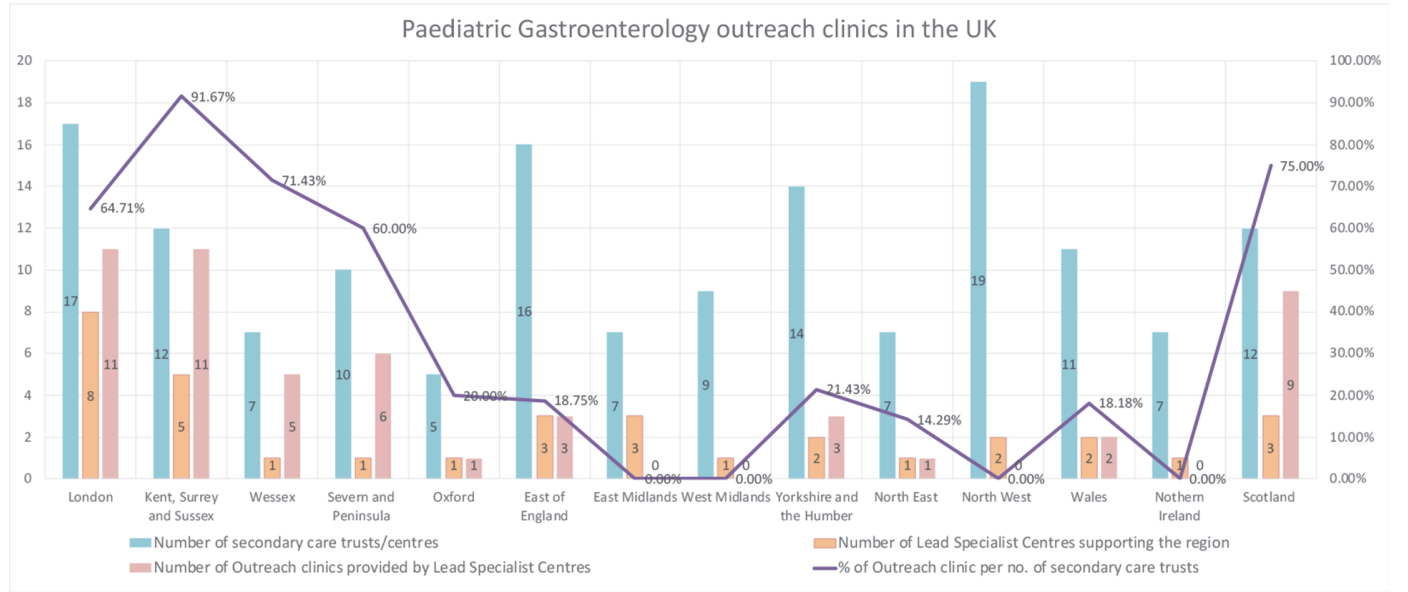

Abstract 010 Figure 2

across the UK. Approximately $62.7 \%$ of these centres had at least one Consultant Paediatrician with SPIN in Gastroenterology. The region with the largest percentage of secondary centres with SPIN doctors was KSS (Kent, Surrey, Sussex) with $91.7 \%$, followed by Scotland with $83.3 \%$. On the other hand, Northern Ireland and the North West of England had the lowest percentages. Southampton, Chelsea and Westminster and Bristol are the LSC which provide the highest number of outreach support. However, nearly a $1 / 3$ of the LSC in the UK do not provide any outreach clinics.

Discussion/Conclusion The results of this pioneering project highlight the wide variance in availability of SPIN doctors and outreach clinics in different regions across the UK. The lack of significant correlation amongst different analysed variables may suggest that this variability is secondary to unquantifiable factors such as geographical reach/constraints, intent and local funding policies. We believe this information is valuable to local, regional and national service commissioning groups in the redirection of efforts and resources to target populations where more urgent intervention is required. Points can be learnt from the top performing regions to improve delivery and establish uniformity of care. This project not only identifies the need for continued work in this domain, but also provides a foundation and structure for further analysis of the current services offered in secondary care.

\section{ACHALASIA CARDIA MANAGEMENT- CHANGING EXPERIENCE OF A TERTIARY PAEDIATRIC GASTROENTEROLOGY CENTRE}

Kwang Yang Lee, Dharamveer Basude. Bristol Royal Hospital for Children

\subsection{6/flgastro-2021-bspghan.11}

Background Achalasia is a primary oesophageal motor disorder of unknown aetiology. Incidence of 0.18/100,000 extrapolates to 23 new presentations per year in the UK. An ESPGHAN Survey revealed that $76 \%$ present to gastroenterology services. Management expertise is shared between paediatric gastroenterologists and paediatric surgeons.

Recent evidence shows that serial pneumatic balloon dilatations (PD) is equally effective compared to Laparoscopic Heller's myotomy with Dor fundoplication (HM) for type 1 and type 2 Achalasia. Peroral Oesophageal Myotomy (POEM) is most effective in Type 3 and resistant Achalasia and is not widely available. This is reflected in American College of Gastroenterology and European Society of Gastrointestinal Endoscopy guidelines. However, few UK paediatric gastroenterology, hepatology and nutrition (PGHAN) centres offer pneumatic balloon dilatations and hence patients may have limited options.

The Bristol PGHAN service has offered Pneumatic balloon dilatation as an option from 2010. Over time, practice has evolved from ad hoc dilatations to serial dilatations with a management algorithm. Families frequently choose PD as it is 